\title{
Decreased Central $\mu$-Opioid Receptor Availability in Fibromyalgia
}

\author{
Richard E. Harris, ${ }^{1}$ Daniel J. Clauw, ${ }^{1}$ David J. Scott, ${ }^{2}$ Samuel A. McLean, ${ }^{3}$ Richard H. Gracely,,${ }^{1}$ and Jon-Kar Zubieta ${ }^{2,4}$ \\ ${ }^{1}$ Department of Internal Medicine, ${ }^{2}$ Department of Psychiatry and Molecular and Behavioral Neuroscience Institute, and Departments of ${ }^{3}$ Emergency \\ Medicine and ${ }^{4}$ Radiology, The University of Michigan, Ann Arbor, Michigan 48109
}

The underlying neurophysiology of acute pain is fairly well characterized, whereas the central mechanisms operative in chronic pain states are less well understood. Fibromyalgia (FM), a common chronic pain condition characterized by widespread pain, is thought to originate largely from altered central neurotransmission. We compare a sample of 17 FM patients and 17 age- and sex-matched healthy controls, using $\mu$-opioid receptor (MOR) positron emission tomography. We demonstrate that FM patients display reduced MOR binding potential (BP) within several regions known to play a role in pain modulation, including the nucleus accumbens, the amygdala, and the dorsal cingulate. MOR BP in the accumbens of FM patients was negatively correlated with affective pain ratings. Moreover, MOR BP throughout the cingulate and the striatum was also negatively correlated with the relative amount of affective pain (McGill, affective score/sensory score) within these patients. These findings indicate altered endogenous opioid analgesic activity in FM and suggest a possible reason for why exogenous opiates appear to have reduced efficacy in this population.

Key words: fibromyalgia; opioid; pain; chronic; positron emission tomography; $\mu$

\section{Introduction}

Sensory perceptions can serve to alert organisms of present and/or future danger. This is particularly evident for the sensation of acute pain. However, neural pain pathways that originally function to warn of potential harm may also become dysfunctional and lead to maladaptive diseased states of a chronic nature (Woolf, 2004). Fibromyalgia (FM), a condition of idiopathic chronic pain, may be one such disorder.

FM is defined on the basis of tenderness and spontaneous chronic widespread pain (Wolfe et al., 1990) and afflicts 2-4\% of individuals in industrialized countries (Wolfe et al., 1995). In addition many FM patients also suffer from psychiatric illnesses such as depression (Giesecke et al., 2003). Unfortunately, because of the lack of readily identifiable peripheral pathology in FM (e.g., muscle or joint inflammation), acceptance of this condition by medical practitioners has been slow (Cohen, 1999).

A growing body of scientific literature suggests that the lack of apparent peripheral pathology in FM might be explained by a primary disturbance in central rather than peripheral pain processing (Clauw and Chrousos, 1997). Data from psychophysical pain testing (Petzke et al., 2003), quantitative EEG (Lorenz et al.,

Received May 7, 2007; revised Aug. 1, 2007; accepted Aug. 2, 2007.

This work was supported by Department of Army Grant DAMD-17/002-0018, Grant M01-RR000042 from the National Center for Research Resources, a component of the National Institutes of Health (NIH), and NIH Grant R01 AT 001415 (J.-K.Z.). R.E.H. was supported by NIH-National Center for Complementary and Alternative Medicine Grant K01 AT01111-01. S.A.M. was supported by NIH Grant K12 RR017607-01. There are no conflicts of interest for any of us with the material presented. We acknowledge V. Napadow and S. Harte for their careful review of this manuscript

Correspondence should be addressed to Dr. Richard E. Harris, Chronic Pain and Fatigue Research Center, 24 Frank Lloyd Wright Drive, P.0. Box 385, Lobby M, Ann Arbor, MI 48106.E-mail: reharris@med.umich.edu. D01:10.1523/JNEUROSCI.2849-07.2007

Copyright $\odot 2007$ Society for Neuroscience $\quad$ 0270-6474/07/2710000-07\$15.00/0
1996), and functional neuroimaging (Gracely et al., 2002; Cook et al., 2004) supports this theory. FM patients display increased neural activations in pain regions such as the insula, the somatosensory cortex, and the cingulate, in response to pressure pain. These same areas are activated in healthy control participants, albeit at higher objective stimulus intensities. Although this suggests that altered pain processing of experimental stimuli occurs in FM, the underlying neurobiology driving clinical symptoms such as pain and depression is unknown.

One potential reason for pain symptoms in FM may be inadequate descending antinociceptive activity. Research suggests that such activity may be deficient or absent in FM (Julien et al., 2005). In humans, the two principal descending inhibitory pain pathways involve either norepinephrine/serotonin or opioids, but psychophysical studies are incapable of distinguishing which of these pathways may be affected. FM patients display low CSF levels of biogenic amines, suggesting a possible deficiency of descending serotonergic/noradrenergic pathways in this condition (Russell et al., 1992). CSF levels of endogenous enkephalins, however, have been noted to be high, which suggests an excess of endogenous opioids in FM (Baraniuk et al., 2004). Although no trials of exogenous opioids in FM have been performed, opioids are not anecdotally found to be useful in treating this and related conditions (Rao and Clauw, 2004). Thus, existing data support a deficit in descending analgesic activity in the serotonergic/noradrenergic system and an overactive opioidergic system; however, as of yet, there is no direct evidence of this.

We used positron emission tomography (PET) to further investigate opioid antinociceptive activity in FM. $\left[{ }^{11} \mathrm{C}\right]$ carfentanil, a selective $\mu$-opioid receptor (MOR) radiotracer, was used to 
assess baseline receptor availability in vivo [binding potential (BP)] in patients and pain-free control participants. We hypothesized that patients with FM may have decreased MOR receptor availability, because they have increased levels of endogenous opioids in the CSF (Baraniuk et al., 2004), possibly leading to receptor downregulation. In addition, we investigated the association of MOR availability with both the affective and sensory dimensions of clinical pain. Finally, as an exploratory analysis, we examined the relationship between MOR availability and depression within FM patients.

\section{Materials and Methods \\ Participants}

As part of an ongoing study investigating the impact of acupuncture treatment in FM, 17 female right-handed patients (age, $44.8 \pm 13.7$ years; duration of FM diagnosis, $8.4 \pm 6.0$ years) were examined with PET. Seventeen right-handed age- and sex-matched control participants (age, $40.4 \pm 11.2$ years) were used as a comparison with the FM group. All analyses were performed on data acquired before acupuncture treatment. Participants gave written informed consent, and the study protocol was approved by the local Institutional Review Board and the Radioactive Drug Research Committee.

All patients (1) met the American College of Rheumatology 1990 criteria (Wolfe et al., 1990) for the diagnosis of FM for at least 1 year; (2) had continued presence of pain $>50 \%$ of days; (3) were willing to limit the introduction of any new medications or treatment modalities for control of FM symptoms during the study; (4) were $>18$ and $<75$ years of age; (5) were female; (6) were right handed; (7) had no alcohol intake $48 \mathrm{~h}$ before PET studies; and (8) were capable of giving written informed consent. Patients were excluded if they (1) had used narcotic analgesics within the past year or had a history of substance abuse; (2) had presence of a known coagulation abnormality, thrombocytopenia, or bleeding diathesis; (3) had the presence of concurrent autoimmune or inflammatory disease, such as rheumatoid arthritis, systemic lupus erythematosus, inflammatory bowel disease, etc., that causes pain; (4) had concurrent participation in other therapeutic trials; (5) were pregnant or nursing mothers; (6) had severe psychiatric illnesses (current schizophrenia, major depression with suicidal ideation, or substance abuse within 2 years); (7) had current major depression; or (8) had contraindications to PET. No patients were taking or had a previous history of opioid medication use. Ten of the FM participants were taking either serotonin reuptake inhibitors or dual serotonin/norepinephrine reuptake inhibitors, whereas seven were not.

All healthy controls were (1) female; (2) right handed; (3) between the ages of 18 and 60; and (4) had no chronic medical illnesses.

\section{Neuroimaging}

Image acquisition. PET scans were acquired with a Siemens (Knoxville, $\mathrm{TN}) \mathrm{HR}^{+}$scanner in three-dimensional mode [reconstructed fullwidth at half-maximum (FWHM) resolution, $\sim 5.5 \mathrm{~mm}$ in-plane and $5.0 \mathrm{~mm}$ axially], with septa retracted and scatter correction. Participants were positioned in the PET scanner gantry, and an intravenous (antecubital) line was placed in the right arm. A light forehead restraint was used to eliminate intrascan head movement. [ $\left.{ }^{11} \mathrm{C}\right]$ carfentanil was synthesized at high specific activity $(>2000 \mathrm{Ci} / \mathrm{mmol})$ by the reaction of $\left[{ }^{11} \mathrm{C}\right]$ methyliodide and a nonmethyl precursor as described previously (Dannals et al., 1985), with minor modifications to improve its synthetic yield (Jewett, 2001); 10-15 mCi (370-555 MBq) were administered during the scan. Receptor occupancy by carfentanil was calculated to be between 0.2 and $0.6 \%$ for brain regions with low, intermediate, and high MOR concentrations, based on the mass of carfentanil administered and the known concentration of opioid receptors in the postmortem human brain (Gross-Isseroff et al., 1990; Gabilondo et al., 1995). Fifty percent of the $\left[{ }^{11} \mathrm{C}\right]$ carfentanil dose was administered as a bolus, and the remaining $50 \%$ was administered by continuous infusion for the remainder of the study. Twenty-eight frames of images were acquired over $90 \mathrm{~min}$ with an increasing duration (30 s up to $10 \mathrm{~min}$ ).
Anatomical magnetic resonance imaging (MRI) scans were acquired in all subjects on a 3 tesla scanner (Signa LX; General Electric, Milwaukee, WI). Acquisition sequences were axial SPGR IR-Prep magnetic resonance (MR) (echo time, $3.4 \mathrm{~ms}$; repetition time, $10.5 \mathrm{~ms}$; inversion time, $200 \mathrm{~ms}$; flip angle, $20^{\circ}$; number of excitations, 1 ; number of contiguous images, 124; thickness, $1.5 \mathrm{~mm}$ ).

Image processing. PET images were reconstructed using iterative algorithms (brain mode; FORE/OSEM, four iterations, 16 subsets; no smoothing) into a $128 \times 128$ pixel matrix in a $28.8 \mathrm{~cm}$ diameter field of view. Attenuation correction was performed through a 6 min transmission scan $\left({ }^{68} \mathrm{Ge}\right.$ source $)$ obtained before the PET study and with iterative reconstruction of the blank/transmission data followed by segmentation of the attenuation image. Small head motions during emission scans were corrected by an automated computer algorithm for each subject before analysis, and the images were coregistered to each other with the same software (Minoshima et al., 1993). Time points were then decay corrected during reconstruction of the PET data. Image data were transformed on a voxel-by-voxel basis into two sets of parametric maps: (1) a tracer transport measure $\left(K_{1}\right.$ ratio $)$ and (2) a receptor-related measure at equilibrium [distribution volume ratio (DVR)]. To avoid the need for arterial blood sampling, the tracer transport and binding measures were calculated using a modified Logan graphical analysis (Logan et al., 1996), using the occipital cortex (an area devoid of MORs) as the reference region. The slope of the Logan plot was used for the estimation of the DVR, a measure equal to the $\left(f_{2} B_{\max } / K_{\mathrm{d}}\right)+1$ for this receptor site and radiotracer. $f_{2} B_{\max } / K_{\mathrm{d}}($ or DVR -1$)$ is the receptor-related measure (BP or MOR availability). The term $f_{2}$ refers to the concentration of free radiotracer in the extracellular fluid and is considered to represent a constant and very small value. $K_{1}$ and DVR images for each experimental period and MR images were coregistered to each other and to the International Consortium for Brain Mapping (ICBM) stereotactic atlas orientation. The accuracy of coregistration and nonlinear warping algorithms was confirmed for each subject individually by comparing the transformed MRI and PET images to each other and the ICBM atlas template.

Group differences were mapped into stereotactic space using $t$ maps of statistical significance with SPM2 (Wellcome Department of Cognitive Neurology, London, UK) and Matlab (MathWorks, Natick, MA) software, with a general linear model. No global normalization was applied to the data, and therefore the calculations presented are based on absolute $f_{2} B_{\max } / K_{\mathrm{d}}$ estimates. Only regions with specific MOR BP were included in the analyses (i.e., voxels with DVR values $>1.1$ ). To compensate for small residual anatomic variations across subjects and to improve signal-to-noise ratios, a three-dimensional Gaussian filter (FWHM, 6 $\mathrm{mm}$ ) was applied to each scan.

Image analysis. The comparisons between patients and control subjects were performed using two-sample $t$ tests, on a voxel-by-voxel basis within SPM2. Significant effects were detected using a statistical threshold that controls a type I error rate at $p=0.05$, corrected for multiple comparisons. These statistical thresholds were estimated using the Euler characteristic (Worsley et al., 1992) based on the number of voxels in the gray matter and image smoothness and the extent of local changes (correction for cluster volume) (Friston et al., 1991). Correlations between MOR BP and the relative amount of the affective quality of clinical pain were made using a regression model on a voxel-by-voxel basis with SPM2. Significant effects were detected using a cluster-corrected threshold $p$ value of 0.05 .

Numerical values for MOR binding were extracted from the image data by averaging the values of voxels contained in the area in which significant effects were obtained in the analyses. These values were then entered into SPSS version 14.0 (SPSS, Chicago, IL) for plotting, to rule out the presence of outliers, and to perform correlations with clinical measures.

Global BP values were also extracted and compared between groups with Student's $t$ test. Because global values were found to be lower in the patients (see Results), global values were used as a covariate in regression analyses in which MOR BP was used as the dependent variable and group status and global BP were independent variables. This allows an estimate of group differences for a specific region, while controlling for differences 
in global scores. To examine effects of concomitant drug usage in the patients, additional regression analyses were performed in which MOR $\mathrm{BP}$ was again used as the dependent variable and clinical pain and drug usage (either taking or not taking reuptake inhibitors; see above) were added as covariates. This final procedure was used to examine the effect of drug usage in the patient group on the relationship between MOR BP and pain.

\section{Clinical assessment}

Clinical pain. Clinical pain was assessed immediately before the PET scan with the Short Form of the McGill Pain Questionnaire (SF MPQ) (Melzack, 1987). The SF MPQ has two subscales that measure "sensory" and "affective" qualities of pain. To assess the relative contribution of the affective dimension of pain, the affective subscore of the SF MPQ was divided by the sensory subscore (affective/sensory). This yields an estimate of the relative contribution of the affective component of pain while controlling for the sensory intensity of the sensation (Petzke et al., 2005). For comparison, we also calculated the ratio of the sensory versus the affective subscores of the SF MPQ (i.e., sensory/affective).

Psychological assessment. Depressive symptoms were assessed with the Center for Epidemiological Studies-Depression Scale (Radloff, 1977). This is a 20 -item self-report instrument that was developed by the National Institute of Mental Health to detect major or clinical depression in adolescents and adults in both clinical and normal populations. The total score was used for correlation with MOR BP.

\section{Results}

As expected, no significant differences were observed between the FM group and the control group with respect to participant age or sex (all $p>0.05$ ). During PET imaging, FM patients exhibited significant reductions in MOR BP compared with controls in four regions: the bilateral nucleus accumbens (NAc; left, $p<$ 0.02 ; right, $p<0.05$; corrected for multiple comparisons), the left amygdala ( $p<0.05$; corrected for multiple comparisons), and the right dorsal anterior cingulate ( $p<0.05$; corrected for multiple comparisons) (Fig. $1 A-C$; Table 1). Global mean MOR BP values were reduced in the patient group $(p<0.01)$. Because a reduction in global BP value could explain the lower BP values within these regions for the FM participants, we performed regression analyses using regional MOR BP values as the dependent variable and group assignment and global BP as covariates. Both the left $(p<0.001)$ and right $(p<0.05)$ nucleus accumbens and the amygdala $(p<0.005)$ showed reduced MOR BP in the patients after controlling for global BP differences. The dorsal anterior cingulate showed a trend toward significance $(p<0.07)$. These data suggest that FM patients have reduced MOR BP within multiple brain regions.

To assess whether drug usage within the FM participants could be responsible for reduced MOR BP values, we examined the mean MOR BP for each of the above regions in FM participants that were either taking or not taking serotonin reuptake inhibitors or dual serotonin/norepinephrine reuptake inhibitors. No differences in BP were detected for any of these regions between patients that were either taking or not taking this class of drugs (all $p>0.35$ ). These analyses suggest that the reduced binding observed in the patients for these regions is not attributable to medication usage.

Within the FM patients, MOR BP binding in the left NAc was negatively correlated with clinical pain ratings in the affective (Fig. 2) (SF MPQ affective score, $r=-0.53 ; p<0.05$ ) but not the sensory (SF MPQ sensory score, $r=-0.13 ; p>0.50$ ) dimension of pain. Drug usage, when added as a covariate, did not significantly alter this relationship (standardized $\beta$ without drug covariate $=-0.44$; with drug covariate, $\beta=-0.45$; significance of
A
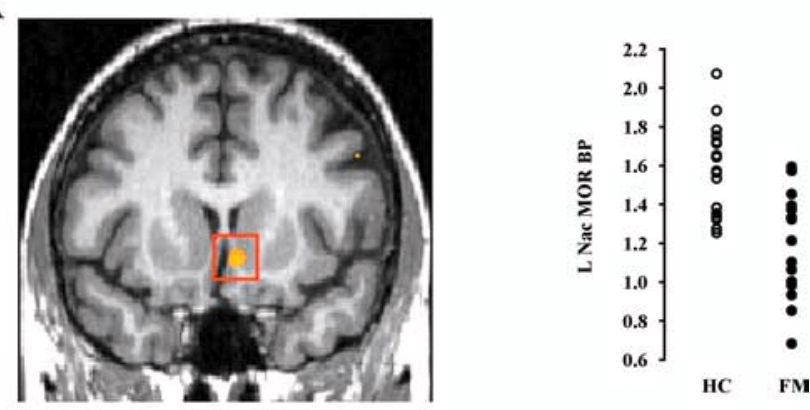

B
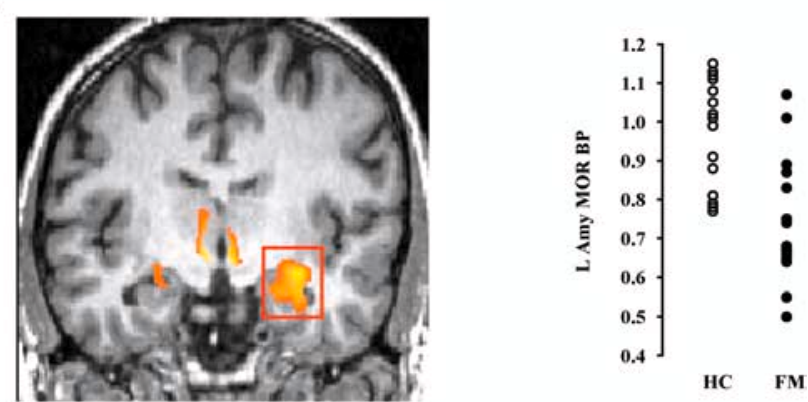

C

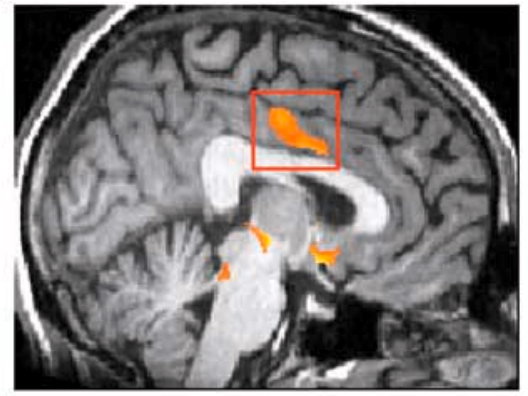

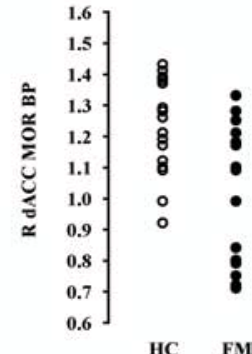

Z score

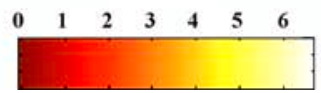

Figure 1. Reduced MOR BP in FM patients. $A-C$, Regions showing reduced MOR BP: left NAC ( $\mathrm{Nac} ; \boldsymbol{A})$, left amygdala (L Amy; $\boldsymbol{B}$ ), and right dACC (R dACC; $\boldsymbol{C}$ ). Plots of individual MOR BP values extracted from PET images are depicted to the right of each corresponding region of interest. FM and healthy control (HC) participants are shown in black and white circles, respectively.

difference, $p=0.59$ ). No statistically significant correlations were observed between clinical pain ratings and the right accumbens, the left amygdala, or the right dorsal anterior cingulate BP of FM patients (all $p>0.05$ ). A significant negative correlation between MOR BP and depressive symptoms was also observed within the amygdala (Table 2).

Because MOR BP within the accumbens was associated with the affective dimension of pain, more so than the sensory dimension, we next investigated the relationship between MOR BP and the relative amount of affective versus sensory pain (SF MPQ, affective score/sensory score). Interindividual differences in MOR binding throughout the cingulate [dorsal anterior (dACC), $p<0.05$; posterior ( $\mathrm{pCC}$ ), $p<0.001$; and, to a lesser extent, anterior ( $\mathrm{aCC}), p=0.09$; all corrected for multiple comparisons] were negatively correlated with the relative amount of affective pain (Fig. $3 A, B$, Table 3 ). Similar findings were detected within the right ventral putamen (Fig. 
Table 1. Regions of reduced $\left[{ }^{11} \mathrm{C}\right]$ carfentanil binding in $\mathrm{FM}$ patients

\begin{tabular}{|c|c|c|c|c|}
\hline Brain region & MNI coordinates $(x, y, z)$ & Z & Cluster size $\left(\mathrm{mm}^{3}\right)$ & $\begin{array}{l}\% \Delta \text { Binding potential } \\
(\mathrm{HC}-\mathrm{FM} ; \text { mean } \pm \mathrm{SD})\end{array}$ \\
\hline $\mathrm{NAc}(\mathrm{I})$ & $9,7,-11$ & $4.1^{*}$ & 159 & $24.7 \pm 15.3$ \\
\hline$N A C(r)$ & $-18,6,-12$ & $3.3^{*}$ & 140 & $18.1 \pm 13.3$ \\
\hline Amygdala (I) & $29,-10,-13$ & $3.7^{*}$ & 269 & $23.7 \pm 14.6$ \\
\hline $\mathrm{dACC}(\mathrm{r})$ & $-4,-11,43$ & $3.1^{*}$ & 182 & $17.7 \pm 12.5$ \\
\hline
\end{tabular}

${ }^{*} p<0.05$, corrected. MNI, Montreal Neurological Institute; I, left; r, right.

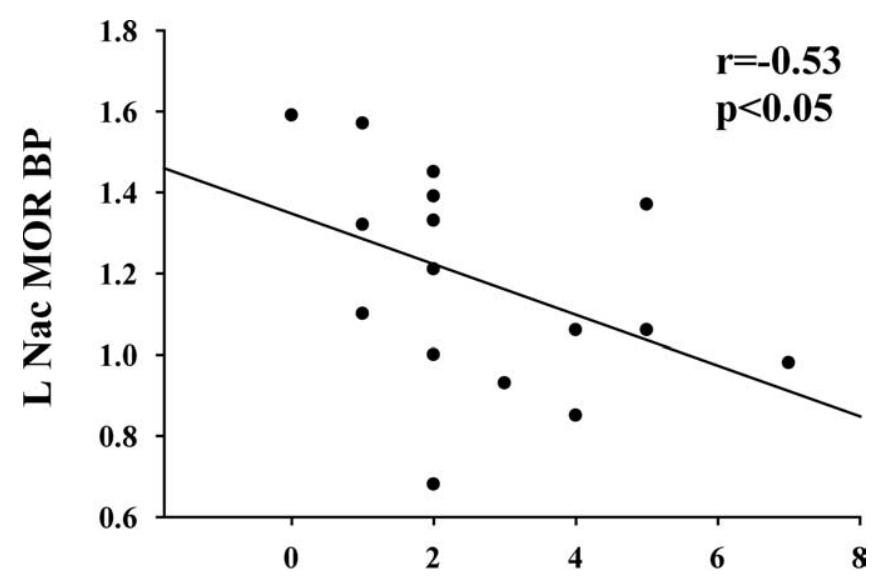

Affective Pain (SF MPQ)

Figure 2. MOR BP is negatively correlated with affective pain. The scatter plot of left accumbens (L Nac) MOR BP and clinical pain (SF MPQ, affective score) reveals a significant negative correlation $(r=-0.53 ; p<0.05)$.

Table 2. Correlations between MOR availability and depression ratings in FM

\begin{tabular}{lc}
\hline Brain region & Rho value \\
\hline $\operatorname{NAC}(I)$ & -0.25 \\
$\operatorname{NAC}(r)$ & 0.16 \\
Amygdala (l) & $-0.49^{*}$ \\
dACC (r) & -0.21 \\
\hline
\end{tabular}

${ }^{*} p<0.05$. I, Left; r, right.

3C). The primary somatosensory cortex and the insula also showed a negative correlation with MOR BP; however, these regions did not reach significance after correction for multiple comparisons $(p>0.05)$. Adding medication usage as a covariate did not significantly alter the relationship between affective/sensory scores and MOR BP for any of these regions (all percentage differences in standardized $\beta$ s with and without covariate $\leq 1.3 \%$; all significance of change, $p>0.20)$. No significant negative correlations were observed between MOR $\mathrm{BP}$ and the relative amount of sensory pain (SF MPQ, sensory score/affective score) within any brain regions (all $p>0.05$ ). No correlations were observed between MOR BP within any of these regions and depressive symptoms (all $p>0.20$ ). These results suggest that in FM patients the affective quality of pain is associated with reduced MOR availability throughout the cingulate and other brain regions commonly associated with pain modulation.

\section{Discussion}

Our data indicate that FM patients have reduced MOR BP within structures typically observed in imaging studies of experimental pain involving healthy control participants. These structures include the amygdala, the cingulate, and the nucleus accumbens.
All of these regions have previously been noted to play some role in nociception and pain. Opioid activity in the nucleus accumbens and the amygdala has been shown to modulate nociceptive neural transmission in animal models of pain (Gear and Levine, 1995; Manning, 1998). Indeed, endogenous opioids play a central role in analgesia and the perception of painful stimuli (Fields, 2004). MOR-mediated neurotransmission in the nucleus accumbens and amygdala has also been shown to be modulated by pain in healthy controls reducing the pain experience (Zubieta et al., 2001), in a manner consistent with animal data. Because the concentration of endogenous opioids is elevated in the CSF of FM patients (Baraniuk et al., 2004), MORs may be highly occupied by endogenous ligand in an attempt to reduce pain or downregulated after prolonged stimulation. Both these effects could explain the reduced MOR BP observed in this study.

An investigation using functional magnetic resonance imaging (fMRI) in FM has associated enhanced neural activity in both the amygdala and the cingulate with depressive symptoms (Giesecke et al., 2005). This further supports the notion that these regions may be involved with evaluating affective aspects of pain and is consistent with our findings of reduced MOR BP within the amygdala and its correlation with depressive symptoms. Indeed, the dorsal anterior cingulate region, identified as having reduced MOR BP in the patients, also showed a negative correlation with the affective dimension of pain (albeit in the opposite hemisphere). These data suggest that MOR availability within the dorsal anterior cingulate is related to the affective dimension of pain. This finding is supported by previous imaging studies of the cingulate (Vogt, 2005).

Two other chronic pain states, rheumatoid arthritis (Jones et al., 1994) and central neuropathic pain following stroke (Jones et al., 2004; Willoch et al., 2004), also display a reduction in opioid receptor BP within the CNS, as measured with the nonselective opioid receptor radiotracer $\left[{ }^{11} \mathrm{C}\right]$ diprenorphine. Although these data may then suggest that reduced opioid receptor availability may be a shared feature across chronic pain states, the regional distribution of reduced receptor binding was dissimilar across these studies and pain conditions. In rheumatoid arthritis pain, reduced opioid receptor binding was observed in the cingulate, frontal, and temporal cortices, whereas for central neuropathic pain, reduced opioid receptor availability was detected primarily within the thalamus, somatosensory cortex, cingulate, and insula. In patients with peripheral neuropathic pain, reduced opioid BP has been observed bilaterally across brain hemispheres, whereas in central neuropathic pain, reductions in BP were observed largely isolated to one hemisphere (Maarrawi et al., 2007). This heterogeneous pattern of reduced binding may reflect different underlying mechanisms operating in these diverse pain conditions. In the case of FM the reductions in MOR BP observed were localized in regions known to be involved in antinociception in animal models (Gear and Levine, 1995; Manning, 

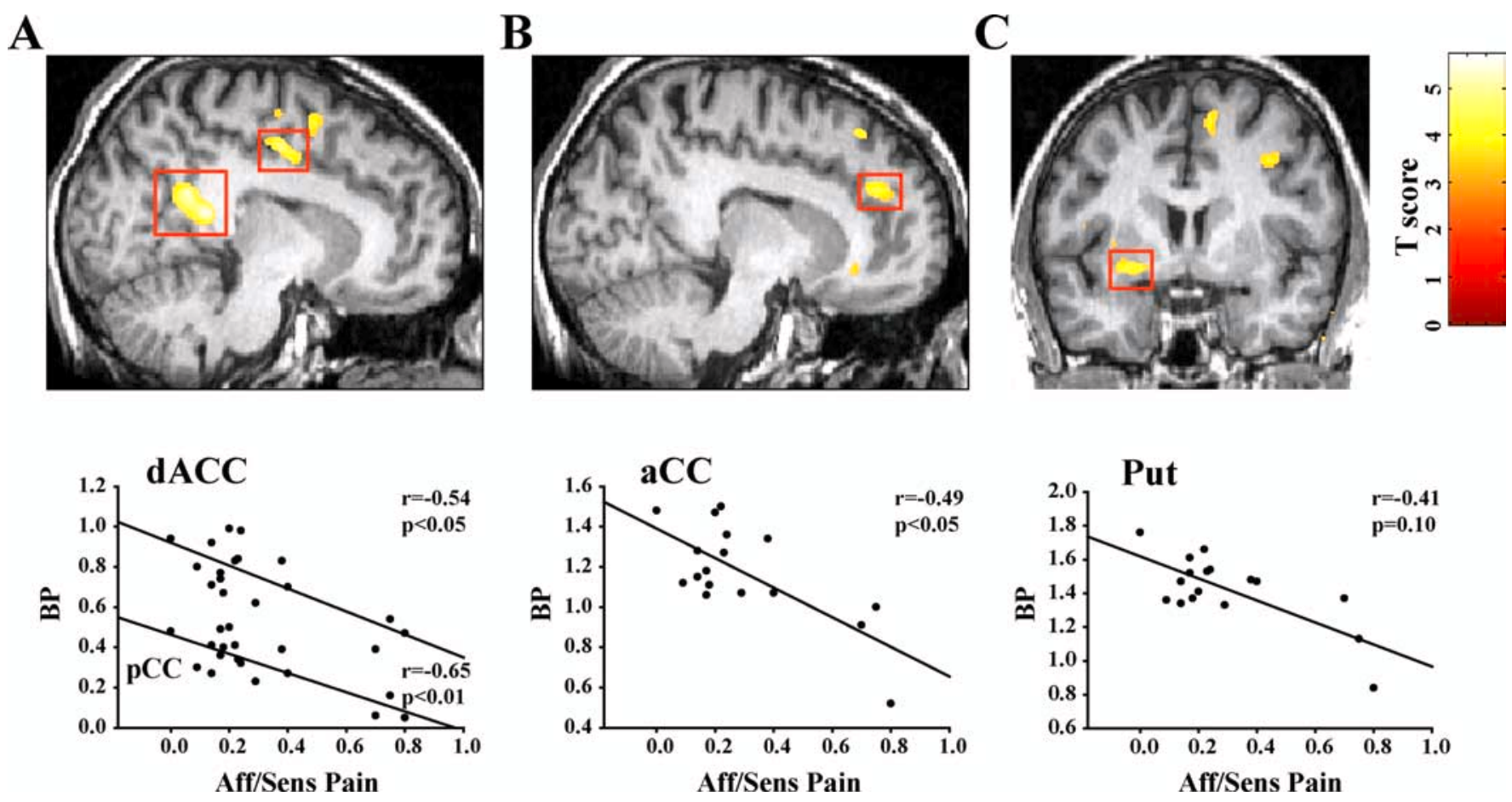

Figure 3. Relative magnitude of the affective pain dimension is associated with reduced MOR BP throughout the cingulate and striatum in FM. $A-C$, Regions showing significant correlations with SF MPQ affective/sensory (Aff/Sens) scores: left dACC and pCC ( $\boldsymbol{A}$ ), right aCC (B), and right putamen (Put; $\boldsymbol{C}$. Scatter plots of individual MOR BP values extracted from PET images are plotted against affective/sensory scores below each image.

Table 3. Regions of correlated MOR BP and relative amount of affective versus sensory pain

\begin{tabular}{lllll}
\hline Brain region & MNI coordinates $(x, y, z)$ & $Z$ & Cluster size $\left(\mathrm{mm}^{3}\right)$ & Rho value for extracted ROls \\
\hline dACC (I) & $10,-11,48$ & $3.75^{* *}$ & 1001 & -0.54 \\
$\mathrm{pCC}(\mathrm{I})$ & $10,-44,17$ & $4.11^{* * *}$ & 936 & -0.65 \\
aCC (r) & $-12,38,28$ & $3.44^{*}$ & 410 & -0.49 \\
Putamen (r) & $-26,4,-8$ & $3.36^{* *}$ & 517 & -0.41 \\
\hline
\end{tabular}

${ }^{*} p=0.09$, corrected; ${ }^{* *} p<0.05$, corrected; ${ }^{* * *} p<0.001$, corrected. MNI, Montreal Neurological Institute; ROls, regions of interest.

1998), as well as pain and emotion regulation, including the affective quality of pain, in humans (Rainville et al., 1997; Zubieta et al., 2001, 2003) (i.e., dorsal anterior cingulate, nucleus accumbens, and amygdala).

Prolonged activations of the MOR by sustained elevations of endogenous agonist have been shown to result in a subsequent decrease in the concentration of MORs in animal models of chronic pain (Li et al., 2005). Chronic administration of morphine may reduce MOR functioning possibly by altering the ability of the receptor to bind to G-proteins, whereas other agonists also downregulate and internalize these receptors (Whistler et al., 1999). If this were the case in FM, sustained activation of MORs by endogenous agonists could ultimately lead to a downregulation of MOR receptor concentration, function, or both. Therefore both mechanisms (i.e., increased release of endogenous opioids and/or a reduction in receptor function) could be responsible for our findings.

We also observed a negative correlation between MOR BP within the accumbens and clinical ratings in the affective dimension of pain. This supports the hypothesis that mechanisms of clinical FM pain are coupled to MOR availability. A strong relationship between pain affect and MOR BP was also observed throughout multiple regions of the cingulate. This is consistent with a proposed role of the dorsal and anterior cingulate in the modulation of pain perception via opioidergic mechanisms (Vogt et al., 1995). Recent investigations of opioid receptor binding in healthy controls showed reduced receptor availability within the rostral cingulate during thermal pain (Sprenger et al., 2006) and sustained muscular pain, which correlated with the suppression of pain affect (Zubieta et al., 2001). Within animal models of experimental pain, microinjection of morphine into the anterior cingulate dosedependently reduced affective components of pain greater than sensory aspects (LaGraize et al., 2006). Our findings of greater affective pain associated with lower MOR BP within the cingulate are consistent with these observations.

We also detected a negative correlation between affective pain and MOR BP values within the posterior cingulate. This is potentially a novel finding because this region is not typically observed in pain imaging trials in humans (Vogt, 2005). However previous trials do suggest that activity within the posterior cingulate, specifically the dorsal aspect, is related to skeletomotor orientation of the body in response to noxious stimuli (Vogt, 2005; Vogt and Laureys, 2005). Because our FM participants experienced clinical pain during the scanning sessions, one could speculate that reduced MOR BP within this region may reflect activation of the endogenous opioid system in an attempt to reduce skeletomotor orientation resulting from spontaneous clinical pain. One additional potential lim- 
itation of this final analysis is that affective and sensory pain dimensions are often highly correlated.

A significant relationship was also detected between MOR availability within the amygdala and depression. Individuals with more depressive symptoms had reductions in MOR BP within the amygdala. This finding is not unexpected, because reduced opioid receptor availability within the amygdala has been previously associated with periods of sadness in patients with major depressive disorder (Kennedy et al., 2006).

Perhaps more important for clinical investigations in FM, our results would predict a lack of efficacy for exogenous opioids in this population. Regardless of whether endogenous opioids are high (Baraniuk et al., 2004) or MORs are downregulated, both scenarios would predict that FM patients would respond less well to exogenous opioids. This prediction awaits future prospective trials of exogenous opioid treatments in FM.

Overall we detect decreased MOR availability in FM patients, demonstrating a dysregulation of this neurotransmitter system in this disease. The reduction in binding was further negatively correlated with affective pain. The observation of specific regional alterations in central opioid neurotransmission in FM suggests that these mechanisms, possibly as a consequence of persistent pain, are involved in the clinical presentation and even the perpetuation of symptoms in this illness. Furthermore, because these receptors are the target of opiate drugs, a profound reduction in the concentration or function of these receptors is consistent with a poor response of FM patients to this class of analgesics, observed anecdotally in clinical settings.

\section{References}

Baraniuk JN, Whalen G, Cunningham J, Clauw DJ (2004) Cerebrospinal fluid levels of opioid peptides in fibromyalgia and chronic low back pain. BMC Musculoskelet Disord 5:48.

Clauw DJ, Chrousos GP (1997) Chronic pain and fatigue syndromes: overlapping clinical and neuroendocrine features and potential pathogenic mechanisms. Neuroimmunomodulation 4:134-153.

Cohen ML (1999) Is fibromyalgia a distinct clinical entity? The disapproving rheumatologist's evidence. Baillieres Best Pract Res Clin Rheumatol 13:421-425.

Cook DB, Lange G, Ciccone DS, Liu WC, Steffener J, Natelson BH (2004) Functional imaging of pain in patients with primary fibromyalgia. J Rheumatol 31:364-378.

Dannals RF, Ravert HT, Frost JJ, Wilson AA, Burns HD, Wagner Jr HN (1985) Radiosynthesis of an opiate receptor binding radiotracer: [11C]carfentanil. Int J Appl Radiat Isot 36:303-306.

Fields H (2004) State-dependent opioid control of pain. Nat Rev Neurosci 5:565-575.

Friston KJ, Frith CD, Liddle PF, Frackowiak RS (1991) Comparing functional (PET) images: the assessment of significant change. J Cereb Blood Flow Metab 11:690-699.

Gabilondo AM, Meana JJ, Garcia-Sevilla JA (1995) Increased density of muopioid receptors in the postmortem brain of suicide victims. Brain Res 682:245-250.

Gear RW, Levine JD (1995) Antinociception produced by an ascending spino-supraspinal pathway. J Neurosci 15:3154-3161.

Giesecke T, Williams DA, Harris RE, Cupps TR, Tian X, Tian TX, Gracely RH, Clauw DJ (2003) Subgrouping of fibromyalgia patients on the basis of pressure-pain thresholds and psychological factors. Arthritis Rheum 48:2916-2922.

Giesecke T, Gracely RH, Williams DA, Geisser M, Petzke F, Clauw DJ (2005) The relationship between depression, clinical pain, and experimental pain in a chronic pain cohort. Arthritis Rheum 52:1577-1584.

Gracely RH, Petzke F, Wolf JM, Clauw DJ (2002) Functional magnetic resonance imaging evidence of augmented pain processing in fibromyalgia. Arthritis Rheum 46:1333-1343.

Gross-Isseroff R, Dillon KA, Israeli M, Biegon A (1990) Regionally selective increases in mu opioid receptor density in the brains of suicide victims. Brain Res 530:312-316.

Jewett DM (2001) A simple synthesis of [11C]carfentanil using an extraction disk instead of HPLC. Nucl Med Biol 28:733-734.

Jones AK, Cunningham VJ, Ha-Kawa S, Fujiwara T, Luthra SK, Silva S, Derbyshire S, Jones T (1994) Changes in central opioid receptor binding in relation to inflammation and pain in patients with rheumatoid arthritis. Br J Rheumatol 33:909-916.

Jones AK, Watabe H, Cunningham VJ, Jones T (2004) Cerebral decreases in opioid receptor binding in patients with central neuropathic pain measured by [11C] diprenorphine binding and PET. Eur J Pain 8:479-485.

Julien N, Goffaux P, Arsenault P, Marchand S (2005) Widespread pain in fibromyalgia is related to a deficit of endogenous pain inhibition. Pain 114:295-302.

Kennedy SE, Koeppe RA, Young EA, Zubieta JK (2006) Dysregulation of endogenous opioid emotion regulation circuitry in major depression in women. Arch Gen Psychiatry 63:1199-1208.

LaGraize SC, Borzan J, Peng YB, Fuchs PN (2006) Selective regulation of pain affect following activation of the opioid anterior cingulate cortex system. Exp Neurol 197:22-30.

Li Z, Proud D, Zhang C, Wiehler S, McDougall JJ (2005) Chronic arthritis down-regulates peripheral mu-opioid receptor expression with concomitant loss of endomorphin 1 antinociception. Arthritis Rheum 52:3210-3219.

Logan J, Fowler JS, Volkow ND, Wang GJ, Ding YS, Alexoff DL (1996) Distribution volume ratios without blood sampling from graphical analysis of PET data. J Cereb Blood Flow Metab 16:834-840.

Lorenz J, Grasedyck K, Bromm B (1996) Middle and long latency somatosensory evoked potentials after painful laser stimulation in patients with fibromyalgia syndrome. Electroencephalogr Clin Neurophysiol 100:165-168

Maarrawi J, Peyron R, Mertens P, Costes N, Magnin M, Sindou M, Laurent B, Garcia-Larrea L (2007) Differential brain opioid receptor availability in central and peripheral neuropathic pain. Pain 127:183-194.

Manning BH (1998) A lateralized deficit in morphine antinociception after unilateral inactivation of the central amygdala. J Neurosci 18:9453-9470.

Melzack R (1987) The short-form McGill Pain Questionnaire. Pain 30:191-197.

Minoshima S, Koeppe RA, Mintun MA, Berger KL, Taylor SF, Frey KA, Kuhl DE (1993) Automated detection of the intercommissural line for stereotactic localization of functional brain images. J Nucl Med 34:322-329.

Petzke F, Clauw DJ, Ambrose K, Khine A, Gracely RH (2003) Increased pain sensitivity in fibromyalgia: effects of stimulus type and mode of presentation. Pain 105:403-413.

Petzke F, Harris RE, Williams DA, Clauw DJ, Gracely RH (2005) Differences in unpleasantness induced by experimental pressure between patients with fibromyalgia and controls. Eur J Pain 9:325-335.

Radloff LS (1977) The CES-D Scale: a self-report depression scale for research in the general population. Applied Psychological Measurement 1:385-401.

Rainville P, Duncan GH, Price DD, Carrier B, Bushnell MC (1997) Pain affect encoded in human anterior cingulate but not somatosensory cortex. Science 277:968-971.

Rao SG, Clauw DJ (2004) The management of fibromyalgia. Drugs Today (Barc) 40:539-554

Russell IJ, Vaeroy H, Javors M, Nyberg F (1992) Cerebrospinal fluid biogenic amine metabolites in fibromyalgia/fibrositis syndrome and rheumatoid arthritis. Arthritis Rheum 35:550-556.

Sprenger T, Valet M, Boecker H, Henriksen G, Spilker ME, Willoch F, Wagner KJ, Wester HJ, Tolle TR (2006) Opioidergic activation in the medial pain system after heat pain. Pain 122:63-67.

Vogt BA (2005) Pain and emotion interactions in subregions of the cingulate gyrus. Nat Rev Neurosci 6:533-544.

Vogt BA, Laureys S (2005) Posterior cingulate, precuneal and retrosplenial cortices: cytology and components of the neural network correlates of consciousness. Prog Brain Res 150:205-217.

Vogt BA, Wiley RG, Jensen EL (1995) Localization of mu and delta opioid receptors to anterior cingulate afferents and projection neurons and input/output model of mu regulation. Exp Neurol 135:83-92.

Whistler JL, Chuang HH, Chu P, Jan LY, von Zastrow M (1999) Functional dissociation of mu opioid receptor signaling and endocytosis: implica- 
tions for the biology of opiate tolerance and addiction. Neuron 23:737-746.

Willoch F, Schindler F, Wester HJ, Empl M, Straube A, Schwaiger M, Conrad B, Tolle TR (2004) Central poststroke pain and reduced opioid receptor binding within pain processing circuitries: a [11C]diprenorphine PET study. Pain 108:213-220.

Wolfe F, Smythe HA, Yunus MB, Bennett RM, Bombardier C, Goldenberg DL, Tugwell P, Campbell SM, Abeles M, Clark P (1990) The American College of Rheumatology 1990 criteria for the classification of fibromyalgia. Report of the Multicenter Criteria Committee. Arthritis Rheum 33:160-172.

Wolfe F, Ross K, Anderson J, Russell IJ, Hebert L (1995) The prevalence and characteristics of fibromyalgia in the general population. Arthritis Rheum 38:19-28.
Woolf CJ (2004) Pain: moving from symptom control toward mechanismspecific pharmacologic management. Ann Intern Med 140:441-451.

Worsley KJ, Evans AC, Marrett S, Neelin P (1992) A three-dimensional statistical analysis for CBF activation studies in human brain. J Cereb Blood Flow Metab 12:900-918.

Zubieta JK, Smith YR, Bueller JA, Xu Y, Kilbourn MR, Jewett DM, Meyer CR, Koeppe RA, Stohler CS (2001) Regional mu opioid receptor regulation of sensory and affective dimensions of pain. Science 293:311-315.

Zubieta JK, Ketter TA, Bueller JA, Xu Y, Kilbourn MR, Young EA, Koeppe RA (2003) Regulation of human affective responses by anterior cingulate and limbic mu-opioid neurotransmission. Arch Gen Psychiatry 60:11451153. 